\title{
Estenosis cervical sintomática por calcificación del ligamento amarillo tras trau- matismo cervical leve
}

\author{
P.D. Delgado-López; V. Martín-Velasco; J.M. Castilla-Díez y *A. Velasco-Oses
}

Servicios de Neurocirugía y *Anatomía Patológica. Hospital General Yagüe. Burgos.

\section{Resumen}

Introducción. La calcificación del ligamento amarillo (CLA) es una rara enfermedad que afecta principalmente a mujeres de edad media o avanzada y de etnia japonesa. Diversas características clínicas y radiológicas la diferencian de la osificación del ligamento amarillo (OLA). Presentamos un caso de estenosis cervical sintomática, aparentemente desencadenado por un traumatismo cervical de carácter leve, en una paciente con una extensa calcificación del ligamento amarillo a nivel cervicodorsal que fue tratada mediante laminectomía descompresiva. Se revisa la literatura al respecto.

Caso clínico. Mujer de 65 años que sufre traumatismo cervical leve como consecuencia de atropello por automóvil. Al año acude al servicio de Neurocirugía refiriendo desde entonces cervicalgia y dolor interescapular que progresivamente habían aumentado en intensidad, sin afectación motora ni sensitiva. En la RMN de columna cervical se objetivó una formación fusiforme epidural posterior de contorno bien delimitado desde C2 a D2, que producía compresión de la médula cervical sin imagen de mielopatía. Se intervino mediante laminectomía descompresiva de los niveles afectados, sin gran mejoría de los síntomas. El estudio anatomopatológico mostró una calcificación extensa del ligamento amarillo.

Discusión. La CLA es un tipo de calcificación distrófica que tiene una etiopatogenia desconocida pero posiblemente relacionada con cambios degenerativos espinales, siendo más frecuente en mujeres y en población japonesa. No se ha descrito una relación causal definitiva entre traumatismo cervical y CLA, aunque es posible que aquél actúe como desencadenante de los síntomas. El paciente tipo con CLA seria una mujer en la séptima década de la vida, que presenta una calcificación preferentemente cervical, de tipo nodular o difuso en la TAC, hipointenso en las secuencias de

Recibido; 16-04-06. Aceptado: 12-07-06 resonancia potenciadas en $\mathrm{T} 1$ y $\mathrm{T} 2$, con mínima captación de gadolinio periférico y con un patrón histológico granular. Histológicamente se diferencia de la OLA por la existencia de hueso maduro únicamente en ésta. Clínicamente puede manifestarse como mielorradiculopatía. El tratamiento quirúrgico de elección en los casos sintomáticos es la laminectomía descompresiva, técnica que, según diversos autores, obtiene excelentes resultados. De confirmarse una relación patogénica entre CLA y traumatismo cervical en futuras observaciones, sería de gran importancia medico-legal pues implicaría el desarrollo de una enfermedad potencialmente grave tras un tipo de traumatismo en principio considerado banal.

PALABRAS CLAVE: Ligamento amarillo. Calcificación distrófica. Laminectomía descompresiva. Traumatismo cervical.

Symptomatic cervical stenosis due to calcification of the ligamentum flavum after mild cervical trauma

\section{Summary}

Introduction. The calcification of ligamentum flavum (CLF) is a rare disease mainly affecting middle or advanced aged Japanese women. Several clinical and radiological features differentiate CLF from the ossification of the ligamentum flavum (OLF). We present a case of symptomatic cervical-dorsal stenosis presenting after mild cervical trauma in a patient with a remarkable CLF who underwent decompressive laminectomy. The literature regarding CLF is reviewed.

Case report. A 65 year-old female suffered mild cervical trauma as a consequence of a car accident. A year later she referred progressive cervical and upper dorsal pain without any sensory or motor defect. Spinal

Abreviaturas. CLA: calcificación ligamento amarillo. CLF: Calcificación ligamentum flavum. OLA: osificación ligamento amarillo. OLF: osificación ligamentum flavum. 
magnetic resonance images showed a well-delineated posterior epidural mass, from C2 to T2, which compressed the spinal cord, without signal of myelopathy. She underwent bilateral laminectomy of the affected levels but no clear improvement occurred. The pathological study of the resected pieces showed a remarkable calcification of the ligamentum flavum.

Discussion. The CLF is a kind of dystrophic calcification of unknown pathogenesis but likely related to the spinal degenerative changes. It usually presents in Japanese females. A definite relation between CLF and cervical trauma has not been established so far, although the latter may possibly trigger the symptoms. Typically, CLF presents in women in the seventh decade, affects preferably the cervical region, it shows nodular or diffuse pattern in the computerized tomography, it is hypointense in TI and T2-weighted MR sequences and exhibits minimum enhancement after gadolinium administration. CLF differentiates from OLF easily by demonstrating the presence of mature bone formation in the latter. Clinically, CLF may present as radiculopathy or myelopathy. The treatment of choice in symptomatic patients is posterior decompression. Excellent results after laminectomy are reported. Future similar observations will be important from a medical-legal point of view if a relation between CLF and cervical trauma is established since CLF may potentially turn into a severe condition.

KEY WORDS: Ligamentum flavum. Dystrophic calcification. Decompressive laminectomy. Cervical trauma.

\section{Introducción}

La calcificación del ligamento amarillo (CLA) en la región cervical es una entidad rara de etiopatogenia incierta que afecta preferentemente a mujeres de etnia japonesa y de edad media y avanzada ${ }^{10,12}$. Actualmente la CLA se distingue de otras patologías similares, como pueda ser la osificación del ligamento amarillo (OLA) en virtud de ciertos datos epidemiológicos e histopatológicos ${ }^{12}$.

Diversas características biomecánicas de la columna cervical, como son la hipermovilidad del segmento medio cervical o la hipertrofia del ligamento amarillo que acompaña a los cambios degenerativos espinales, se han citado como factores predisponentes para el desarrollo de CLA ${ }^{7,11,12}$. En ocasiones, se ha especulado sobre la posibilidad de que un traumatismo de suficiente energía provocara la formación de un hematoma epidural espinal cervical, que idealmente pudiera calcificarse y constituir una condición patológica indistinguible de la CLA, tanto desde el punto de vista de la anatomía radiológica como en su forma de presentación clínica, usualmente como radiculomielopatía. Asimismo,

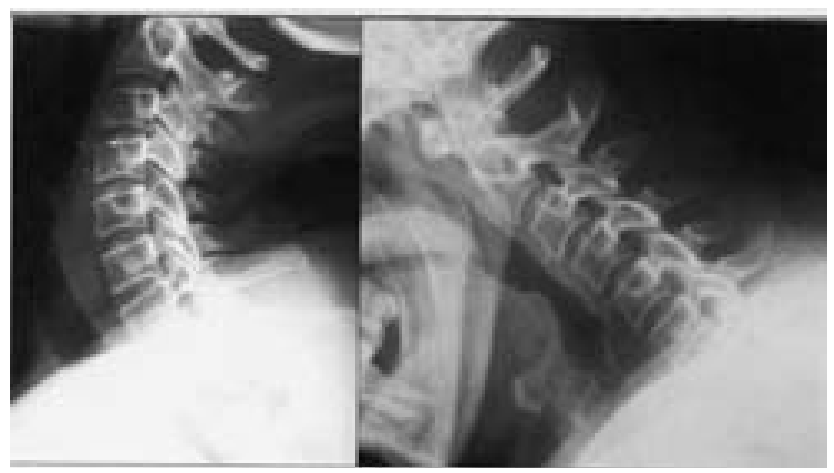

Figura 1. Imágenes de rayos $x$ de columna cervical en flexión y extensión, que muestran una motilidad y estabilidad conservadas.

se ha descrito la aparición de un cuadro de mielopatía aguda tras un traumatismo leve en un paciente con osificación del ligamento amarillo ${ }^{6}$. Histopatológicamente, la CLA se diferencia de forma sencilla de la OLA por la presencia de hueso maduro, únicamente en esta última. Sin embargo, hasta la fecha, la literatura no aclara cuál es la historia natural y las características clínico-patológicas de un supuesto hematoma epidural cervical postraumático calcificado.

Presentamos un caso de estenosis cervical sintomática, aparentemente desencadenado por un traumatismo cervical de carácter leve, en una paciente en la que se evidenció una extensa calcificación del ligamento amarillo a nivel cervicodorsal epidural posterior que fue tratada mediante laminectomía descompresiva y extirpación del ligamento engrosado. Así mismo, se revisan y discuten las características clínicas e histopatológicas de esta enfermedad.

\section{Caso clínico}

Mujer de 65 años de edad, sin antecedentes patológicos de interés, que en Julio de 2003 sufrió un atropello por automóvil siendo diagnosticada de traumatismo cervical leve. La paciente fue tratada de forma sintomática con medicación antiinflamatoria y collarín cervical blando durante tres semanas. Tras doce meses acude al servicio de Neurocirugía de nuestro centro refiriendo sensación de mareo continuo, cervicalgia posterior y dolor interescapular que habían aumentado progresivamente en intensidad hasta resultar incapacitantes. La paciente explícitamente relacionaba el inicio de los síntomas con el momento preciso del accidente. En la exploración neurológica no se evidenció afectación motora ni sensitiva de miembros; únicamente contractura bilateral de la musculatura cervical dorsal y de trapecios. Las radiografías cervicales mostraron una correcta movilidad en flexo-extensión de las columnas cervical y dorsal, sin evidencia de inestabilidad segmentaria (figura 1). En la RMN de columna cervical se 


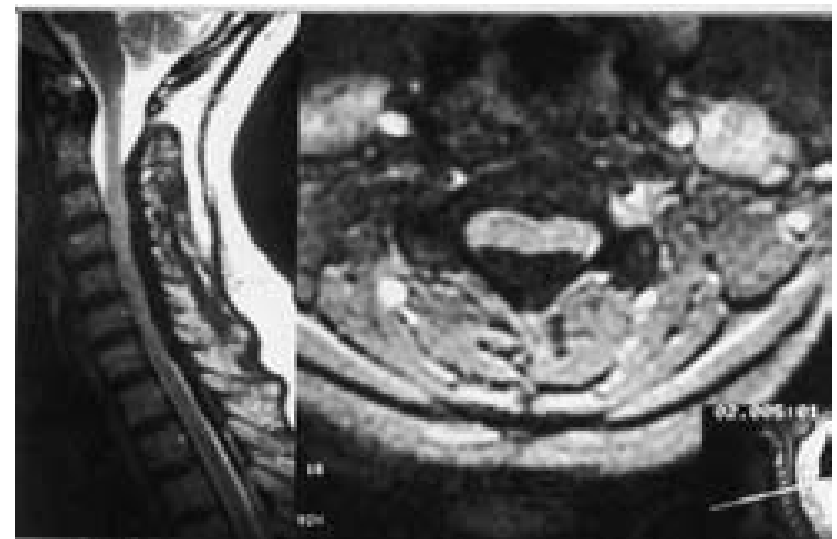

Figura 2. Imagen de RM potenciada en T2 preoperatoria. Extensa formación epidural posterior cervicodorsal hipointensa que impronta el saco tecal.

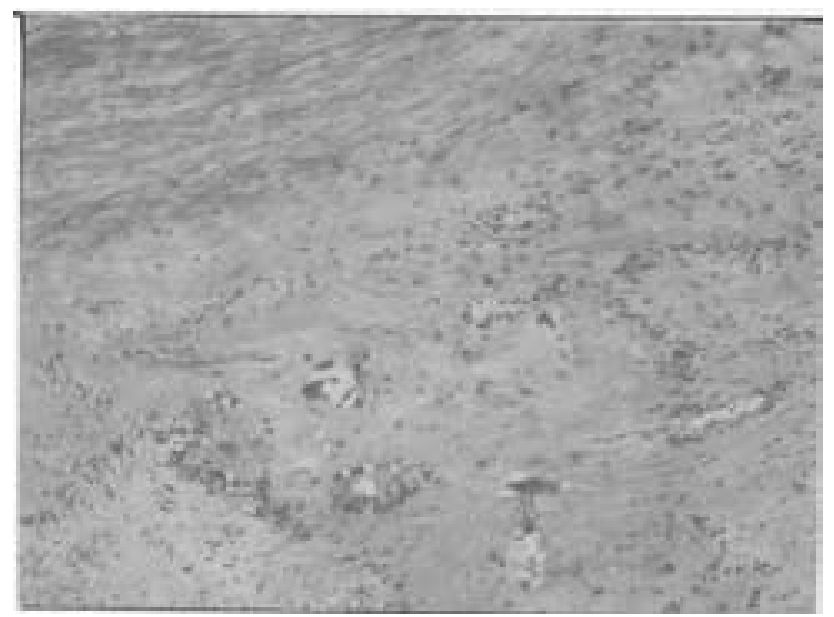

Figura 3. Ligamento amarillo normal en la porción superior y depósitos cálcicos azulados rodeados por histiocitos en empalizada en la porción inferior.

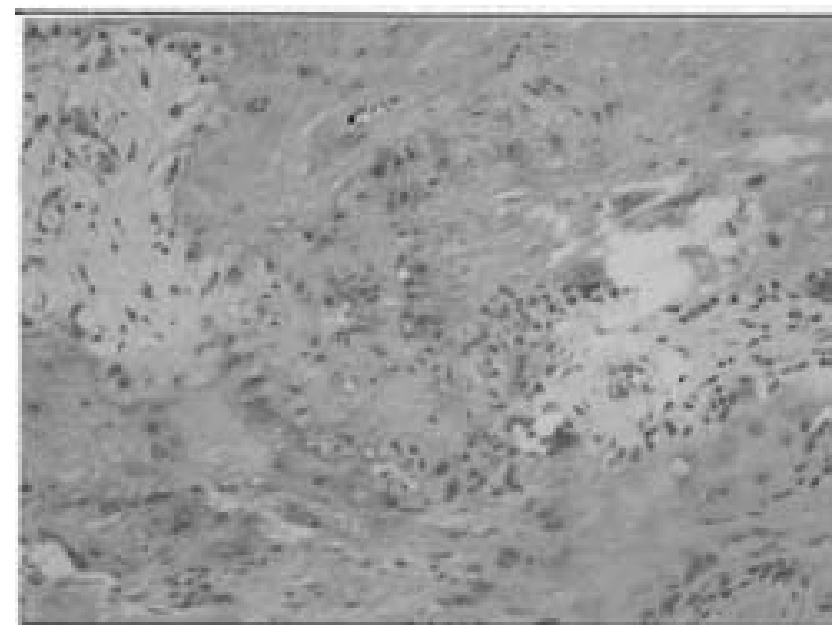

Figura 4. Detalle del material cálcico e histiocitos cercanos formando granulomas inflamatorios de tipo cuerpo extraño.

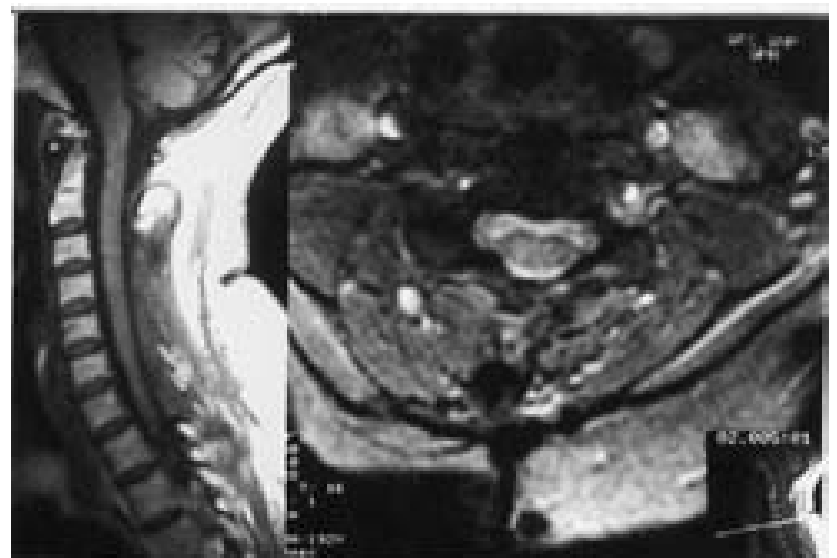

Figura 5. Imagen RM potenciada en T1 postoperatoria. Adecuada descompresión del canal medular.

objetivó una lesión ocupante de espacio localizada en la región cervico-dorsal epidural posterior, a expensas de una formación fusiforme de contorno bien delimitado que se extendía desde C2 hasta D2, hipointensa en las secuencias potenciadas en T1 y T2, correspondiente a la localización anatómica del ligamento amarillo. Dicha formación producía impronta sobre la médula cervical a nivel de su borde posterior, fundamentalmente en el segmento C6-C7, sin imagen de mielopatía asociada (figura 2).

Ante el empeoramiento progresivo del dolor y su imposibilidad de control medicamentoso, se propuso la realización de descompresión quirúrgica. En Julio de 2004, la paciente aceptó ser intervenida realizándose laminectomía descompresiva cervical de los niveles afectados. No se produjeron complicaciones postoperatorias, aunque tampoco un alivio significativo del dolor. Histológicamente, las muestras de ligamento amarillo mostraban múltiples calcificaciones distróficas (figura 3 ) y reacción inflamatoria granulomatosa de tipo cuerpo extraño con abundantes histiocitos en empalizada (figura 4). Tras 19 meses de seguimiento, la paciente refiere persistencia del dolor cervical posterior, de menor intensidad, mal controlable con medicación analgésica, y sin afectación neurológica evidenciable. El estudio de RM postoperatorio muestra una correcta alineación vertebral y una adecuada descompresión del saco tecal (figura 5). Actualmente, la paciente se encuentra en proceso de litigio para la obtención de compensación económica y declaración de invalidez como consecuencia del accidente.

\section{Discusión}

El caso presentado ejemplifica una rara enfermedad que, si bien está documentada en la literatura, fundamentalmente por autores japoneses, pertenece al poco conocido capítulo de las calcificaciones de ligamentos 
vertebrales, cuyo exponente más estudiado es la osificación del ligamento longitudinal posterior ${ }^{13}$. La localización cervicodorsal añade, así mismo, un componente de rareza y de dificultad de manejo a este caso.

Recientemente, Ugarriza y $\operatorname{cols}^{12}$ han delineado de forma precisa las características clínico-patológicas que diferencian a los pacientes afectos de CLA frente a los que presentan OLA. Según estos autores, el paciente tipo con CLA sería una mujer en la séptima década de la vida, que presenta una calcificación preferentemente cervical, de tipo nodular o difuso en la TAC, hipointenso en las secuencias de resonancia potenciadas en T1 y T2, con mínima captación de gadolinio periférico y con un patrón histológico granular. Por el contrario, el paciente con OLA puede ser varón o mujer de menor edad que el anterior, con osificación nodular o en forma de "V" visible en la TAC, idéntico comportamiento radiológico en la RM, preferentemente localizado en la región torácica baja, en el que se verifica histológicamente la formación de hueso maduro y que puede asociarse a osificación del ligamento longitudinal posterior. La aposición de pirofosfato cálcico dihidratado e hidroxiapatita puede aparecer en cualquier paciente afecto de CLA, y evidenciarse a través de un análisis mediante difracción de rayos $\mathrm{x}^{14}$.

Las características del caso descrito se ajustan estrechamente a las de una CLA en una mujer de etnia no japonesa.

La etiopatogenia de la CLA es todavía desconocida y se han postulado factores biomecánicos, hormonales y raciales en su formación ${ }^{4,10}$. Probablemente, la CLA sea un proceso de desarrollo lento y progresivo en cuya presentación clínica podrían influir factores metabólicos, inflamatorios, degenerativos y traumáticos ${ }^{6,12}$. Hasta la fecha no se ha establecido una relación causal definitiva entre el traumatismo cervical y la formación de CLA. En el caso descrito, a pesar de la coincidencia temporal del inicio de los síntomas con el momento del accidente, es difícil apoyar la explicación del traumatismo como causa de la calcificación; parece razonable, sin embargo, considerar el traumatismo cervical, en apariencia leve, como el desencadenante de la cervicalgia sobre una CLA previamente desarrollada. Ya con anterioridad se ha descrito un caso de síndrome de Brown-Sequard subagudo causado por una hernia discal C5-C6 sobre la base de un canal cervical estenótico debido a una extensa CLA cervical ${ }^{12}$. Así mismo, Li y cols ${ }^{6}$ reportan un caso de OLA con mielopatía de presentación aguda tras un traumatismo menor.

Clínicamente, la CLA cervical suele presentarse como un cuadro insidioso de mielopatía con defectos sensitivos, motores o mixtos, con especial predilección por los niveles cervicales medios, frecuentemente en los segmentos C5 y C6. La patología degenerativa espinal, mucho más prevalente que la CLA, suele acompañar y complicar el cuadro

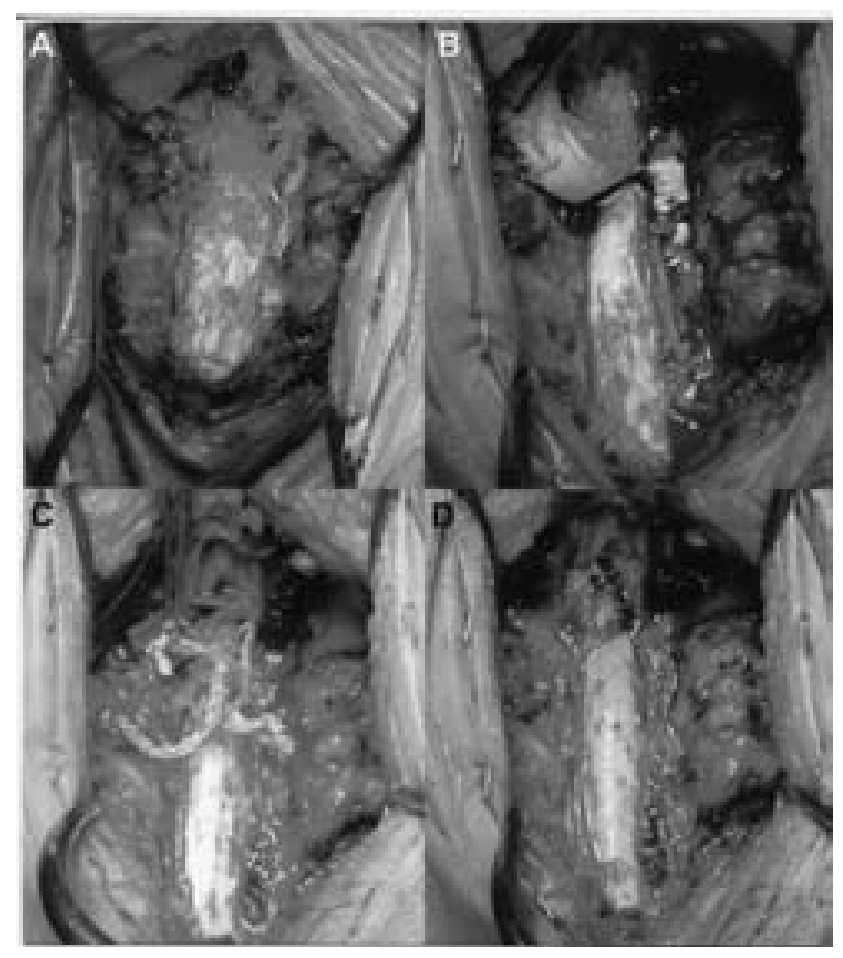

Figura 6. Imágenes intraoperatorias. A: Laminectomía y exposición del ligamento calcificado. B: Extracción del material cálcico engrosado de gran dureza que formaba parte del ligamento amarillo. C: Filamentos de ligamento amarillo engrosado y calcificado durante la resección. D: Duramadre expuesta y liberada de la compresión ligamentosa suprayacente.

clínico, especialmente en aquellos pacientes que presentan estenosis crítica del canal cervical. Se han descrito en la literatura casos de CLA que afectan tanto a mujeres japonesas (lo más frecuente) como a individuos de raza negra y caucásicos ${ }^{2}$. Aunque está ampliamente documentada la asociación epidemiológica entre etnia japonesa y CLA, se desconocen los determinantes concretos que relacionan a ambas.

Histológicamente, se describen casos de CLA tanto por depósito de cristales de pirofosfato cálcico dihidratado (pseudogota) como por cristales de hidroxiapatita o por una combinación de ambos ${ }^{3,9.12,14}$. Kubota y $\operatorname{cols}^{5}$ han estudiado detenidamente la ultraestructura de los depósitos cálcicos entre las fibras de colágeno y elastina del ligamento amarillo y cómo éstos, eventualmente, coalescen para formar los nódulos calcificados visibles al microscopio óptico. Por otra parte, Baba y cols ${ }^{1}$ sugieren que la CLA cervical podría simplemente ser la manifestación espinal de una enfermedad sistémica por depósito de cristales de calcio. En nuestro caso, las fibras de ligamento amarillo presentaban nódulos calcificados y una reacción inflamatoria similar a la reacción a cuerpo extraño. 
Desde el punto de vista terapéutico no parece ser relevante la distinción entre CLA y OLA, pues en ambos casos la modalidad terapéutica recomendada es la descompresión del canal medular. La laminectomía cervical ha sido la técnica quirúrgica empleada por diversos autores con anterioridad ${ }^{1,3,7,10,12,14}$ con resultados prácticamente siempre satisfactorios. Es interesante destacar que el íntimo contacto (incluso la auténtica fusión) entre el ligamento amarillo y la duramadre dificulta su separación durante el acto quirúrgico, de tal forma que, en ocasiones, ha sido necesario realizar una extirpación de ambos en bloque y colocación de duroplastia ${ }^{12}$. Haraguchi y cols ${ }^{3}$ reportan un caso en el que resecaron en bloque la masa del ligamento calcificada junto a las láminas de C5 y C6, fragmento que, sin embargo, no estaba en continuidad con la duramadre. En nuestro caso dichas estructuras pudieron ser separadas, el ligamento calcificado fue resecado en láminas fibrosas de gran dureza y la duramadre quedó finamente adelgazada pero sin solución de continuidad (figura 6).

Optamos por la técnica de la laminectomía medial estrecha sin artrodesis posterior, a pesar de la extensión craneocaudal de la misma, respetando las facetas articulares y la inserción de la musculatura cervical en C2. La realización de laminoplastia cervical expansiva o laminectomía cervical acompañada de fijación posterior son técnicas que pretenden minimizar las probabilidades de desarrollar una futura deformidad cifótica. La elección de la técnica quirúrgica es una cuestión sujeta a debate, en la que la decisión obliga a sopesar, por un lado, la morbilidad quirúrgica de una fijación cervicodorsal extensa y la limitación del movimiento cervical que produce, y por otro, el riesgo de desarrollo de cifosis a largo plazo. Estos riesgos no están claramente cuantificados en la literatura. Se debe tener en cuenta que la presencia de material metálico de fijación interna provoca artefacto en la imagen de resonancia magnética, lo que dificulta el seguimiento por imagen de las lesiones mielopáticas.

Diversas circunstancias que acompañan al caso descrito sugieren la posibilidad de que, en la mala evolución de la paciente, hayan influido factores que pudieran estar relacionados con la posibilidad de obtener compensación económica. El hecho de que la paciente achaque de forma inequívoca el inicio de sus síntomas con el momento del traumatismo cervical (aun tan alejado en el tiempo como un año antes); que dicho traumatismo se deba a un accidente de vehículo a motor (atropello); que presente síntomas vagos y de difícil control referidos al cuello y hombros; que no se haya producido apenas mejoría con la descompresión quirúrgica; y que se encuentre pendiente de obtención de declaración de invalidez en ausencia de deficiencias neurológicas, apoyan una posible motivación rentista. Esta situación está ampliamente documentada en otras patologías, como el esguince (latigazo) cervical, en las que frecuentemente confluyen circunstancias similares ${ }^{8}$.

El caso presentado aporta evidencia limitada y controvertida en cuanto a la relación entre traumatismo cervical leve y desarrollo de CLA. De confirmarse esta relación patogénica en futuras observaciones, estaríamos frente a una condición clínica de enorme importancia médico-legal pues establecería un precedente en cuanto a desarrollo de secuelas tardías provocadas por un tipo de traumatismo muy frecuente y habitualmente considerado banal.

El desarrollo de una CLA cervical en una mujer de etnia no japonesa, diagnosticada en el contexto de un traumatismo cervical leve, que afecta de forma extensa a prácticamente toda la columna cervical y charnela cervicodorsal, y cuya sintomatología no se ha resuelto de forma satisfactoria tras la descompresión por abordaje posterior, hacen de éste un caso original que ayuda a comprender mejor el espectro clínico de esta rara enfermedad.

\section{Agradecimientos}

A Eva María Corrales García por la lectura cuidadosa, corrección del manuscrito y ayuda con la iconografía.

\section{Bibliografía}

1. Baba, H., Maezawa, Y., Kawahara, N., Tomita, K., Furusawa, N., Imura, S.: Calcium crystal deposition in the ligamentum flavum of the cervical spine. Spine 1993; 18: 2174-2181.

2. Hankey, G.J., Khangure, M.S.: Cervical myelopathy due to calcification of the ligamentum flavum. Aus N Z J Surg 1988; 58: 247-249 (abstract).

3. Haraguchi, K., Yamaki, T., Kurokawa, Y., Ohtaki, M., Ibayashi, Y., Ueda, T., y cols.: A case of calcification of the cervical ligamentum flavum. No Shinkei Geka 1996; 24: 6973 (abstract).

4. Iwasaki, Y., Akino, M., Abe, H., Tsuru, M., Tashiro, K., Miyasaka, K., y cols.: Calcification of the ligamentum flavum of the cervical spine. Report of four cases. J Neurosurg 1983; 59: 531-534.

5. Kubota, T., Kawano, H., Yamashima, T., Ikeda, K., Hayashi, M., Yamamoto, S.: Ultrastructural study of calcification process in the ligamentum flavum of the cervical spine. Spine 1987; 12: 317-323.

6. Li, K.K., Chung, O.M., Chang, Y.P., So,Y.C.: Myelopathy caused by ossification of ligamentum flavum. Spine 2002; 27: E308-312.

7. Muthukumar, N., Karuppaswami, U.: Tumoral calcium pyrophosphate dehydrate deposition disease of the ligamentum flavum. Neurosurgery 2003; 53: 103-108.

8. Nachemson, A., Jonsson, E.: Neck and back pain. The scientific evidence of causes, diagnosis and treatment. Philadelphia, Lippincott Williams \& Wilkins, 2000. 
9. Pascal-Moussellard, H., Cabre, P., Smadja, D., Kaidomar, S., Catonne Y.: Myelopathy due to calcification of the cervical ligamenta flava: a report of two cases in West Indians patients. Eur Spine J 1999; 8: 238-240.

10. Sato, K, Hayashi, M., Kubota, T., Kawano, H., Handa, Y., Kabuto, M.: Symptomatic calcification and ossification of the cervical ligamentum flavum: clinical, radiological and pathological features. Br J Neurosurg 1989; 3: 597-602.

11. Stollman, A., Pinto, R., Benjamin, V., Kricheff, L.: Radiologic imaging of symptomatic ligamentum flavum thickening with and without ossification. AJNR Am J Neuroradiol 1987; 8: 991-994.

12. Ugarriza, L.F., Cabezudo, J.M., Porras, L.F., Rodríguez-Sánchez, J.A.: Cord compression secondary to cervical disc hemiation associated with calcification of the ligamentum flavum: case report. Neurosurgery 2001; 48:673-676.

13. Vasudevan, A., Knuckey, N.W.: Ossification of the ligamentum flavum. J Clin Neurosci 2002; 9: 311-313.

14. Yamagami, T., Kawano, N., Nakano,H.: Calcification of the cervical ligamentum flavum. Case report. Neurol Med Chir (Tokyo) 2000; 40: 234-238.

Delgado-López, P.D.; Martin-Velasco, V.; Castilla-Díez, J.M.; Velasco-Oses, A.: Estenosis cervical sintomática por calcificación del ligamento amarillo tras traumatismo cervical leve. Neurocirugía 2007; 18: 141-146.

Correspondencia postal: Pedro David Delgado López. Servicio de Neurocirugía. Hospital General Yagüe. Avenida Cid, 96. 09005 Burgos. 